\title{
RSS based Vertical Handoff algorithms for Heterogeneous wireless networks - A Review
}

\author{
Abhijit Bijwe \\ Electronics \& Comm. Engg. Deptt \\ P.I.E.T.Nagpur
}

\author{
Dr. C.G.Dethe \\ Electronics \& Telecom Engg. Deptt \\ Principal, \\ P.I.E.T. Nagpur
}

\begin{abstract}
Heterogeneous networks are integrated in fourth generation. To have seamless communication and mobility between these heterogeneous wireless access networks, support of vertical handoff is required. Vertical handover is convergence of heterogeneous networks for e.g.:- handover between WLAN and cellular networks.

In this paper, three algorithms on RSS based vertical handoff are discussed. First, algorithm is adaptive lifetime based vertical handoff, which combines RSS and estimated lifetime (expected duration after which the MT will be able to maintain its connection with WLAN) to decide the vertical handover. Second algorithm, is based on dynamic RSS threshold which is more suitable for handover from WLAN to 3G network. Third algorithm is a traveling distance prediction method, which works well for WLAN to cellular networks and vice versa. This avoids unnecessary handoff and also minimizes failure probability.
\end{abstract}

Keywords- RSS; WLAN; 3G; VHD.

\section{INTRODUCTION}

Convergence of heterogeneous network is getting a lot of attention. To be precise, in $4 \mathrm{G}$ network, a mobile terminal incorporated with multiple interfaces will be able to choose the appropriate available access links. In 4G systems, handoff management is more complex, as it covers not only horizontal handoff but also vertical handoff. In horizontal handoff, where an MT moves between two different cells or access points within the same wireless communication system. While in vertical handover, MT moves from one wireless system to another wireless system, for example, from cellular network to wireless LAN system. In this paper, we do not address the horizontal handoff, as traditional RSS based algorithms which works good to support the horizontal handoff . RSS based handoff algorithm is generally applied to homogeneous network and can be extended to heterogeneous network. Numerous Vertical handover decision algorithms are proposed in various research papers which takes into account several parameters such as Bandwidth, Power consumption, Cost, Security etc. Based on these parameters, cost function algorithm and multiple attribute decision algorithms may be used. These algorithms use different set of parameters [5] to provide better handoff.

Some of the problems associated with these algorithms are

1) Too many parameters may affect the performance of VHD algorithms and relationship between these parameters is very complicated, therefore, how to select those most important parameters as decision factors, and also take into account the effects of other parameters, so that handoff performance can be guaranteed and proper performance tradeoff can be achieved is an important \& difficult problem.

2) MADM algorithms are the most challenging ones because of their pre training requirements. Hence it suffers from longest handover delay. Also the system is complex.

RSS based algorithms are less complex and can be used between macro cellular and microcellular networks.

High handover failure probability is observed for algorithm without inclusion of RSS.

In this paper, we will be focusing on various Mathematical Models in RSS based vertical handover decision algorithms.

We make an attempt to provide a comparative analysis of three RSS based vertical handoff algorithms.

\section{RSS BASED VHD ALGORITHMS}

In this, the handoff decisions are made by comparing RSS (received signal strength) of the current network with the preset threshold values. These algorithms are less complex and may be combined with other parameters such as bandwidth, cost etc. to have a better handover decisions. We describe here three RSS based algorithms in the following sections.

\section{A. ALIVE-HO (adaptive lifetime based vertical handoff) algorithm. -}

Zahran, Chen and Sreenan [6]proposed algorithm for handover between 3G Networks and WLAN by combining the RSS with an estimated life time (duration over which the current access technology remains beneficial to the active applications ). ALIVE-HO always uses an uncongested network whenever available. It continues using the preferred network (i.e. WLAN) as long as it satisfies the QoS requirements of the application.

Two different vertical handoff scenarios are discussed: Moving out of the preferred network (MO) and Moving in to the preferred network (MI), where the preferred network is usually the underlay network that provides better and economical service. Hence, extending the utilization of the WLAN, as long as it provides satisfactory performance is the main considerations of vertical handoff algorithm design.

We observe the method through the following scenarios. 
In first scenario, when the MT moves away from the coverage area of a WLAN into a $3 \mathrm{G}$ cell, a handover to the $3 \mathrm{G}$ network is initiated. The handover is done under the conditions that

(a) RSS average of the WLAN falls below predefined threshold. (MO threshold) and (b) the estimated life time is atleast equal to the required handoff signaling delay. The MT continuously calculate the RSS mean using the moving average method.[4]

$$
\overline{R S S}[\mathrm{~K}]=\frac{1}{W a v} \sum_{i=0}^{W a v-1} \overline{R S S}[k-i]
$$

Here $\overline{R S S}[\mathrm{k}]$ is RSS mean at time instant $\mathrm{k}$, and $\mathrm{W}_{\mathrm{av}}$ is the window size, a variable that changes with velocity of the velocity of mobile terminal. Then, the lifetime metric EL [k] is calculated by using $\overline{R S S}[\mathrm{k}]$, ASST Application signal strength threshold), S[k],RSS change rate.

$$
\mathrm{EL}[\mathrm{k}]=\frac{\overline{R S S}[\mathrm{k}]-\mathrm{ASST}}{\mathrm{S}[\mathrm{k}]}
$$

ASST (Application signal strength threshold) chosen to satisfy the requirements of the active applications. S [K] represents RSS decay rate.In second scenario, when the MT moves towards a WLAN cell, the handover to the WLAN is done if the average RSS is larger than MI Threshold. WLAN and the available bandwidth of the WLAN meet the bandwidth requirement of the application. Table given below shows lost frames during the handoff transition area for the received stream.

\begin{tabular}{|l|l|l|l|l|l|l|}
\hline ASST (in dBs) & -90 & -89 & -88 & -87 & -86 & -85 \\
\hline $\begin{array}{l}\text { Lost } \\
\text { frames_100kbit/s }\end{array}$ & 13.3 & 5 & 3 & 0.67 & 0 & 0 \\
\hline $\begin{array}{l}\text { Lost } \\
\text { frames_300kbit/s }\end{array}$ & 38 & 28 & 4 & 0.33 & 0 & 0 \\
\hline
\end{tabular}

TABLE 1.1 FRAMES LOST CORRESPONDING TO ASST

Based on the obtained results and subjective testing, the optimal value for UDP based streaming is chosen as $-86 \mathrm{~dB}$.

By introducing $E L[k]$ the algorithm adapts to the application requirements and reduces unnecessary handovers. Second, there is an improvement on the average throughput for user because MT prefers to stay in WLAN cell as long as possible.

However packet delay grows, due to the critical fading impact near the cell edges, which may result in severe degradation in the user perceived QoS. This phenomenon results in a tradeoff between improving the system resource utilization and satisfying the user Qos requirements. This issue can be critical for delay sensitive applications and degrade their performance. ASST is tuned according to various system parameters, including delay thresholds, MT velocities, handover signaling costs and packet delay penalties.

\section{B. Algorithm on Adaptive RSS Threshold}

Mohanty and Akyildiz[2] in paper "A cross-layer ( Layer 2 +3 ) Handoff Management Protocol" proposed a WLAN to $3 \mathrm{G}$ handover decision method. In this method, RSS of current network is compared with dynamic RSS threshold $\left(\mathrm{S}_{\mathrm{th}}\right)$ when MT is connected to a WLAN access point. We observe the following notations with reference to fig 1.1 which shows a handoff from current network (AP) referred as WLAN, to the future network (BS), referred as $3 \mathrm{G}$.

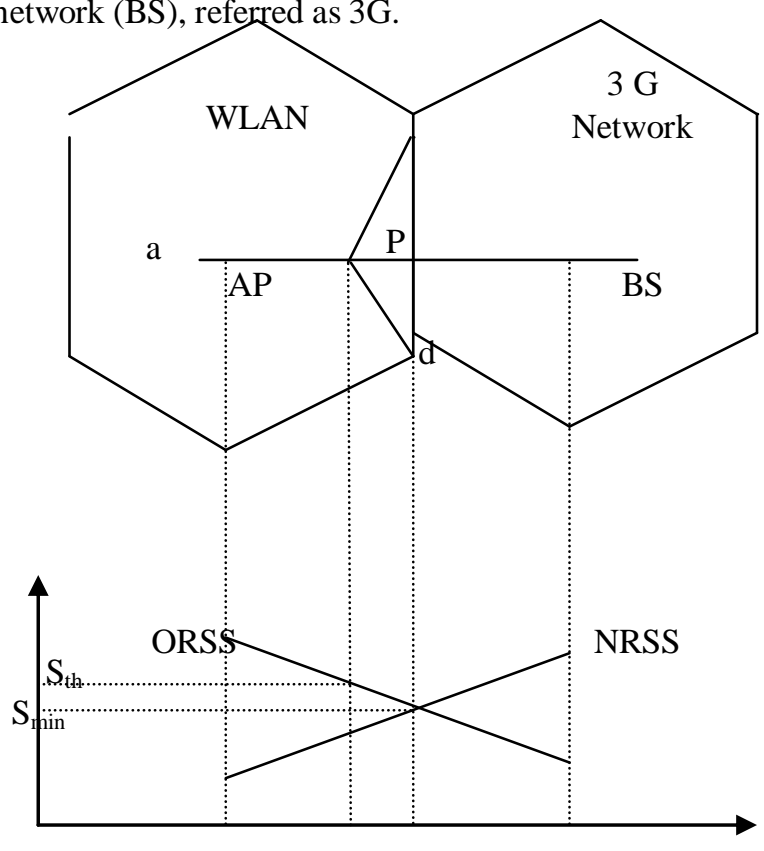

Fig 1.1 Analysis of handoff process

* $\mathrm{S}_{\text {th }}$ : The threshold value of the RSS to initiate the handover process. Therefore, when the RSS of WLAN referred to as ORSS(old RSS) in fig drops below $S_{\text {th }}$, the registration procedures are initiated for MT's handover to the 3G network.

$*$ a :The cell size we assume that the cells are of hexagonal shape.

$\mathrm{d}$ : is the shortest distance between the point at which handover is initiated and WLAN boundary. We observe the Path loss Model [1] given by

$$
\mathrm{P}(\mathrm{x})=\operatorname{Pr}\left(\mathrm{d}_{0}\right)\left(\frac{d_{0}}{x}\right)^{\alpha}+\varepsilon
$$

Where $\mathrm{x}$ is the distance between the AP and MT, and $\mathrm{P}_{\mathrm{r}}\left(\mathrm{d}_{0}\right)$ is the received power at a known reference distance $\left(\mathrm{d}_{0}\right)$. The typical value of $\mathrm{d}_{0}$ is $1 \mathrm{~km}$ for macrocells, $100 \mathrm{~m}$ for outdoor microcells, and $1 \mathrm{~m}$ for indoor picocells.

The numerical value of $\mathrm{P}_{\mathrm{r}}\left(\mathrm{d}_{0}\right)$ depends on different factors, such as frequency, antenna heights, and antenna gains, $\alpha$ is the path loss exponent. The typical values of $\alpha$ ranges from 3 to 4 and 2 to 8 for macrocellular and microcellular environment. 
$\varepsilon \quad$ - is a Zero mean Gaussian random variable that represents the statistical variation in $\mathrm{P}_{\mathrm{r}}(\mathrm{x})$ caused by Shadowing. Typical std. deviation of $\varepsilon$ is $12 \mathrm{~dB}$. scenario.

We observe the path loss model applied to the

$$
\begin{gathered}
\mathrm{P}_{\mathrm{r}}(\mathrm{a}-\mathrm{d})=\mathrm{P}_{\mathrm{r}}(\mathrm{a})\left(\frac{a}{a-d}\right)^{\alpha}+\varepsilon \\
\mathrm{P}_{\mathrm{r}}(\mathrm{a}-\mathrm{d})=\mathrm{P}_{\mathrm{r}}(\mathrm{a})+10 \alpha \log _{10}\left(\frac{a}{a-d}\right)+\varepsilon \\
\mathrm{S}_{\text {th }}=\mathrm{S}_{\text {min }}+10 \alpha \log _{10}\left(\frac{a}{a-d}\right)+\varepsilon
\end{gathered}
$$

When the MT is located at point $\mathrm{P}$, the assumption is that it can move in any direction with equal probability, i.e. the pdf of MT's direction of motion $\theta$ is

$\mathrm{f}_{\theta}(\theta)=\frac{1}{\Pi-(-\Pi)}=\frac{1}{2 \Pi} \quad-\Pi<\theta<\Pi$

As per assumption, that MT's direction of motion and speed remains the same from point $\mathrm{P}$ until it moves out of the coverage area of WLAN. As the distance of P from WLAN boundary is not very large, this assumption is realistic.

The need for handoff to cellular network arises only if MTs direction of motion from $\mathrm{P}$ is in the range [ $\theta \varepsilon$ $\left.\left(-\theta_{1}, \theta_{1}\right)\right]$

Where $\theta_{1}=\arctan \left(\frac{a}{2 d}\right)$, otherwise the handoff initiation is false. The probability of false handoff initiation is

$$
\mathrm{P}_{\mathrm{a}}=1-\int_{-\theta_{1}}^{\theta_{1}} f_{\theta}(\theta) d \theta
$$

$\mathrm{P}($ unfavourable event $)=1-\mathrm{P}($ favourable event $)$

$$
\begin{aligned}
& =1-\frac{1}{2 \Pi}\left(2 \theta_{1}\right) \\
& =1-\frac{1}{\Pi} \arctan \left(\frac{a}{2 d}\right)
\end{aligned}
$$

When the direction of motion of MT from $\mathrm{P} \beta \varepsilon$ $\left[\left(-\theta_{1}, \theta_{1}\right)\right]$ the time it takes to move out of the coverage area of WLAN cell is given by

$$
\text { time }=\frac{\text { dis } \tan c e}{\text { speed }}
$$

From fig $\operatorname{Cos} \beta=\frac{d}{x}$

$$
\begin{aligned}
& \operatorname{Sec} \beta=\frac{x}{d} \quad, \mathrm{x}=\mathrm{d} \sec \beta \\
& \text { Hence } \mathrm{t}=\frac{x}{v} \\
& \mathrm{t}=\frac{d \sec \beta}{v} \quad \ldots . . . . . . .(3)
\end{aligned}
$$

Pdf of $\beta$ is

$$
\begin{aligned}
& \mathrm{f}_{\beta}(\beta)=\left\{\frac{1}{2 \theta_{1}}-\theta_{1}<\beta<\theta_{1}\right. \\
& \begin{cases}0 & \text { otherwise }\end{cases}
\end{aligned}
$$

From (3), $\mathrm{t}$ is a function of $\beta$ i. e. $\mathrm{t}=\mathrm{g}(\beta)$ in $\left[-\theta_{1}, \theta_{1}\right]$

$$
g(\beta)=\frac{d \sec \beta}{v}
$$

Therefore pdf of $\mathrm{t}$ is given by

$$
f_{t}(t)=\sum_{i} \frac{f_{\beta\left(\beta_{i}\right)}}{g^{\prime}\left(\beta_{i}\right)}
$$

Where $\beta_{\mathrm{i}}$ are the roots of equation $\mathrm{t}=\mathrm{g}(\beta)$ in [ $\left.-\theta_{1}, \theta_{1}\right]$

And for each of these roots

$$
\begin{aligned}
& \mathrm{f}_{\beta}\left(\beta_{\mathrm{i}}\right)=\frac{1}{2 \theta_{1}} \quad \text { for } \mathrm{i}=1 \text { and } 2 \\
& \mathrm{f}_{t}(t)=\frac{1}{2 \theta_{1}\left|g^{\prime}\left(\beta_{i}\right)\right|}+\frac{1}{2 \theta_{1}\left|g^{\prime}\left(\beta_{i}\right)\right|} \\
& \mathrm{f}_{t}(t)=\frac{1}{\theta_{1}\left|g^{\prime}\left(\beta_{i}\right)\right|}
\end{aligned}
$$

where $\mathrm{g}^{\prime}(\beta)$ is derivative of $\mathrm{g}(\beta)$ given by

$$
\begin{aligned}
\mathrm{g}^{\prime}(\beta) & =\frac{d \sec \beta \tan \beta}{v} \\
& =\frac{d \sec \beta\left(\sqrt{\sec ^{2} \beta}-1\right)}{v} \\
& =\frac{v t\left(\sqrt{\left(\frac{v t}{d}\right)^{2}-1}\right)}{v}
\end{aligned}
$$


$\mathrm{g}^{\prime}(\beta)=\mathrm{t} \sqrt{\frac{v^{2} t^{2}}{d^{2}}} \quad-1 \quad$ from (3) .....7

Using (6) \& (7), the pdf of $t$ is given by

$$
\mathrm{f}_{t}(t)=\left\{\frac{d}{\theta_{1} t \sqrt{v^{2} t^{2}-d^{2}}, \frac{d}{v}<t<\frac{\sqrt{\frac{a^{2}}{4}+d^{2}}}{v}}\right\}
$$

The probability of handoff failure is given by

$$
\begin{aligned}
& \mathrm{P}_{\mathrm{f}}=\left\{\begin{array}{l}
1 \\
1
\end{array}>\sqrt{\frac{a^{2}}{4}+d^{2}}\right. \\
& \left\{\mathrm{P}(\mathrm{t}<\tau) \frac{d}{v}<\tau<\frac{\sqrt{\frac{a^{2}}{4}+d^{2}}}{v}\right. \\
& \left\{\quad 0 \quad \tau \leq \frac{d}{v}\right. \\
& \tau \text { - Handoff signaling delay }
\end{aligned}
$$

$$
\text { when } \frac{d}{v}<\tau<\frac{\sqrt{\frac{a^{2}}{4}+d^{2}}}{v} \operatorname{using}(8)
$$$$
\mathrm{P}(\mathrm{t}<\tau) \quad=\quad \int_{0}^{\tau} f_{t}(t) d t
$$

$$
\begin{array}{ll}
=\quad \int_{\frac{d}{v}}^{\tau} \frac{d}{\Pi t \sqrt{v^{2} t^{2}}-d^{2}} d t \\
=\quad \int_{\frac{d}{v}}^{\tau} \frac{d}{\Pi d t \sqrt{\frac{v^{2} t^{2}}{d^{2}}-1} d t} \\
=\quad \int_{\frac{d}{v}}^{\tau} \frac{1}{\frac{v}{d} \cdot \frac{d}{v} \sqrt{\frac{v^{2} t^{2}}{d^{2}}-1}} d t \\
\approx \frac{1}{\theta_{1}} \arccos \left(\frac{d}{v t}\right)
\end{array}
$$

Now using (9) \& (10)

$$
\begin{aligned}
& \mathrm{P}_{\mathrm{f}}=\left\{1 \quad \tau>\frac{\sqrt{\frac{a^{2}}{4}}+d^{2}}{v}\right. \\
& \left\{\frac{1}{\theta_{1}} \arccos \left(\frac{d}{v \tau}\right) \frac{d}{v}<\tau<\frac{\sqrt{\frac{a^{2}}{4}+d^{2}}}{v}\right. \\
& \{0 \ldots \ldots \ldots \ldots \ldots . . . . .(8) \tau \\
& \mathrm{P}_{\mathrm{f}}=\frac{\arccos \left(\frac{d}{v \tau}\right)}{\arctan \left(\frac{d}{v \tau}\right)} \\
& \mathrm{P}_{\mathrm{f}}=\frac{\frac{\Pi}{2}-\frac{d}{v \tau}}{\frac{\Pi}{2}-\frac{2 d}{\sqrt{4 d^{2}+a^{2}}}}
\end{aligned}
$$

The use of adaptive RSS threshold helps reducing the handoff failure probability and also reducing unnecessary handovers. The exact value of $S_{t h}$ will depend on MT's speed and handoff signaling delay at a particular time. Adaptive $S_{\text {th }}$ is used to limit handoff failure. However, in this algorithm, the handover from $3 \mathrm{G}$ network to a WLAN is not efficient when MTS traveling time inside a WLAN cell is less than the than the handover delay. This may lead to wastage of network resources.

\section{A Traveling Distance Prediction Based Method.}

To minimize unnecessary handover over Mohanty's Method. Yan et al[3] developed VHD algorithm that takes into consideration the time the mobile terminal is expected to spend within a WLAN cell. A handover to a WLAN is initiated if the WLAN coverage is available and the estimated traveling time. Inside the WLAN cell is larger than the time threshold.

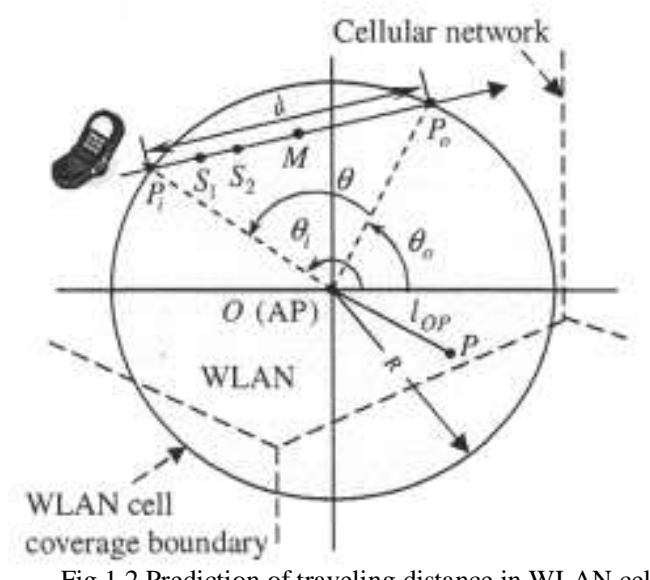

Fig 1.2 Prediction of traveling distance in WLAN cell 
Assume that the MT starts receiving a sufficiently strong signal at point $\mathrm{P}_{\mathrm{i}}$ and the signal strength drops below the usable level at point $\mathrm{P}_{0}$.

Angles $\theta \mathrm{i}$ and $\theta_{0}$ are both uniformly distributed in $[0,2 \Pi]$ where $\theta=\theta \mathrm{i}-\theta_{0}$

The probability density function (pdf) of $\theta$ as fallows

$\mathrm{f}_{\theta}(\theta)=\frac{1}{\Pi}\left(1-\frac{\theta}{2 \Pi}\right), 0 \leq \theta \leq 2 \Pi$

By replacing 1 with $\theta$, d with $2 \Pi 1$ ranges from 0 to $\mathrm{d}$

From the geometric configuration in fig

$$
\begin{aligned}
& \operatorname{Sin} \frac{\theta}{2}=\frac{d / 2}{R} \\
& \operatorname{Sin} \frac{\theta}{2}=\frac{d}{2 R} \\
& \operatorname{Sin}^{2} \frac{\theta}{2}=\left(\frac{d}{2 R}\right)^{2} \\
& \operatorname{Sin}^{2} \frac{\theta}{2}=\frac{d^{2}}{4 R^{2}} \\
& 2 \operatorname{Sin}^{2} \frac{\theta}{2}=\frac{d^{2}}{2 R^{2}} \\
& 2 \mathrm{R}(1-\operatorname{Cos} \theta)=\mathrm{d}^{2} \\
& \mathrm{~d}^{2}=2 \mathrm{R}^{2}(1-\cos \theta)
\end{aligned}
$$

The Pdf of $d$ is expressed as from (3) \& (4)

$$
\mathrm{f}_{\mathrm{D}}(\mathrm{d})=\frac{2}{\Pi \sqrt{4 R^{2}-d^{2}}} \quad 0 \leq \mathrm{d} \leq 2 \mathrm{R}
$$

The cdf of $\mathrm{d}$ can be derived by integrating

$$
\begin{array}{ll}
\mathrm{f}_{\mathrm{D}}(\mathrm{d})=\frac{2}{\Pi} \frac{1}{\sqrt{1-\left(\frac{d}{2 R}\right)^{2}}} & \\
\mathrm{P}(\mathrm{d} \leq D)=\left\{\frac{2}{\Pi} \operatorname{Sin}^{-1}\left(\frac{D}{2 R}\right)\right. & , 0 \leq \mathrm{D} \leq 2 \mathrm{R} \\
1 & 2 \mathrm{R}<\mathrm{D}
\end{array}
$$

We observe a distance threshold parameter $\mathrm{L}$ whichwill be used to make handover decisions. Whenever the estimated traveling distance $d$ is greater than $\mathrm{L}$, the MT will initiate the handover procedures. L may be calculated by using spanning algorithm.

$$
\begin{aligned}
& \mathrm{P}=\frac{2}{\Pi}\left[\operatorname{Sin}^{-1}\left(\frac{v \tau_{i}}{2 R}\right)-\operatorname{Sin}^{-1}\left(\frac{L}{2 R}\right), 0 \leq L \leq v \tau\right] \\
& \left\{\begin{array}{l}
0 \quad v \tau<L
\end{array}\right.
\end{aligned}
$$

Thus the value of $\mathrm{L}$ for a maximum tolerable failure or unnecessary handover probability as

$$
\mathrm{L}=2 \mathrm{R} \operatorname{Sin}\left(\operatorname{Sin}^{-1}\left(\frac{v \tau}{2 R}\right)-\frac{\Pi}{2} P\right.
$$

The time threshold ( $\mathrm{T}_{\text {WLAN }}$ ) is calculated as

$$
\begin{aligned}
& \mathrm{T}_{\text {WLAN }}=\frac{2 R}{v} \operatorname{Sin}\left(\operatorname{Sin}^{-1}\left(\frac{v \tau}{2 R}\right)-\frac{\Pi}{2} P\right) \\
& \mathrm{P} \rightarrow \text { is maximum tolerable handover failure or } \\
& \text { unnecessary handover probability. }
\end{aligned}
$$

$\tau$ is the handover delay from cellular network to WLAN. In this method, VEPSD algorithm can be used to estimate $\mathrm{v}$ and $\tau$ respectively

The traveling time $\left(\mathrm{t}_{\mathrm{WLAN}}\right)$

$$
\mathrm{t}_{\mathrm{WLAN}}=\frac{R^{2}-l o s^{2}+v^{2}\left(t_{s}-t_{\text {in }}\right)^{2}}{v^{2}\left(t_{s}-t_{\text {in }}\right)}
$$

Where $\mathrm{R} \rightarrow$ radius of WLAN Cell,

$1_{\mathrm{os}} \rightarrow$ distance between access point and where the MT takes RSS.sample.

$\mathrm{t}_{\mathrm{s}} \& \mathrm{t}_{\mathrm{in}} \rightarrow$ are the times at which RSS sample is taken and MT enters the WLAN cell coverage respectively.

$\mathrm{t}_{\mathrm{WLAN}}>\mathrm{T}_{\mathrm{WLAN}} \rightarrow$ handover is initiated.

Even though the speed of the MT increases, the probabilities remain in the same. For higher speeds, our method yields lower probability of handover failures and unnecessary handover than the Mohanty's Method.

But the method relies on sampling and averaging RSS points, which introduces increased handover delay.

\section{CONCLUSION}

As per the discussion above, we conclude that Adaptive lifetime based method gives an Improvement in average throughput for user because MT prefers to stay in WLAN cell. But, packet delay grows near edges of the WLAN cell due to fading of signal which results in degradation of Qos. To solve this issue ASST is tuned according to various parameters such as delay thresholds, MT velocities, handover signaling costs and packet delay penalties. Adaptive RSS threshold algorithm works good for handover from WLAN to $3 \mathrm{G}$ network .It helps in reducing handoff failure probability and also reducing 
unnecessary handover between WLAN to $3 \mathrm{G}$ as dynamic RSS threshold is dependent on MTs speed and handoff signaling delay. This algorithm is not efficient when handover is from $3 \mathrm{G}$ to WLAN, if traveling time inside WLAN cell is less than the handover delay. For this case traveling distance prediction based method works fine.

These algorithms minimize unnecessary handover for handover from $3 \mathrm{G}$ to WLAN. But the method relies on sampling and averaging RSS points which introduce increased handover delay. But, the sampling of RSS periodically will eliminate the assumption of MTs speed being fixed in WLAN cell.

\section{FUTURE DIRECTIONS}

A improvement to the scheme is to periodically sample the RSS, recalculate and refine the estimations for $v$ to improve the performance, and eliminate the assumption that the MTS speed remains fixed inside the WLAN cell. Based on the application and economic point of view ( i.e cost ) the handover decision inside the WLAN Cell can be taken. User can be given the choice of selecting the network depending on the factors such as cost or critical application which requires cellular network.

\section{REFERENCES}

[1] T. S. Rappaport, Wireless Communications: Principles and Practice.Prentice Hall, July 1999.
[2] S. Mohanty and I. F. Akyildiz, "A cross-layer (Layer $2+3$ ) handover management protocol for next-generation wireless systems," IEEE Trans. Mobile Computing, vol. 5, pp. 1347-1360, Oct. 2006.

[3] Xiaohuan Yan,N,Nallasamy Mani, and Y.Ahme S, ekercio`glu, “A Traveling Distance Prediction Based Method to minimize Unnecessary Handovers from Cellular Networks to WLANs," IEEE communication letters, vol. 12, pp. 14-16, 2008.

[4] Ahmed H. Zahran and Ben Liang "Performance Evaluation Framework for Vertical Handoff Algorithms in Heterogeneous Networks",in :Proceedings of the 2005 IEEE International Conference on Communications(ICC05),Seoul,Korea,May2005. pp.173-178

[5] Xiaohuan Yan,.Ahmet S, ekercio glu, Sathyanarayan "A Survey of vertical decision algorithms in fourth generation heterogeneous networks"Elsevier,2010, pp.1848-1863

[6] A. H. Zahran and B. Liang, "ALIVE-HO: Adaptive lifetime vertical handoff for heterogeneous wireless networks," Technical Report, University of Toronto.

\section{AUTHORS PROFILE}

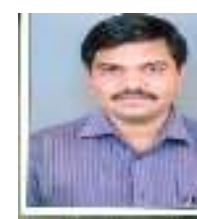

Abhijit Bijwe is $\mathrm{PhD}$ student at the Department of Electronics \& Communication engineering at Nagpur university.Has received B.E.from Amravati university and received M.E. from Mumbai university.His current research area is vertical handover algorithm in heterogeneous networks.

Dr.C.G.Dethe has done Doctrate from Amravati University. Has done B.E. \& M.E. from Amravati and Nagpur University. Currently, He is Principal in Priyadarshini Institute of Engineering \& Technology, Nagpur. His research area is Measurement of Traffic in Mobile Networks. He is guiding $10 \mathrm{PhD}$ students. 